\title{
The coordinate system transformation of a serial kinematic structures and use in the derivation of systems motion equations
}

\author{
Jiří Zátopek ${ }^{1, a}$ \\ ${ }^{1}$ Tomas Bata University in Zlín, Faculty of Applied Informatics, Nad Stráněmi 4511, 76005 Zlín, Czech Republic
}

\begin{abstract}
This text discusses the use of transformation matrices to determine the motion equations of the complex mechanical structure. Use of the transformation matrix does not apply only to motion equations but has the general use in relative positions determine of objects in the 3D space. Analysed model is divided into seven physical objects, the transformation matrix and the corresponding inertia/pseudo-inertia matrix is included in each of them. This matrices are strictly necessary to the system dynamic description using the matrix form of Lagrange Equations of the Second Type. Another possibility to use the transformation matrix is shown in the camera system measurement. Model was designed in 3D CAD system SolidWorks, MATLAB was used for the mathematical calculations.
\end{abstract}

\section{Introduction}

Derivation of systems motion equations (deductive identification) is a crucial part of designing the motion control law of any mechanical structure. These structures are composed of mass objects and acts by not inconsiderable effect on other components of the system.

Each mass object has inertia in the motion. These inertias are reflected in the form of centrifugal/centripetal and Coriolis generalized forces with the combination of rotational movements - non-linearity into the system is introduced. The whole system has, therefore, highly nonlinear behaviour during fast movements - non-linearities are bounded to the rate of change of state variables largely.

The system which is the object of our interest is called "Ball and plate", shown in Figure 1. To derive the equations of motion of the system the transformation matrices individual parts are advantageous to know. These transformation matrices can be further used for derivation of the inertia matrix, the position determining of the individual parts of the assembly in a 3D space, and non-measurable state variables obtaining - during the whole process procedure. The matrix form of Lagrange equations of the second type there will be used, which involves the use of the transformation matrices directly.

The Denavit-Hartenberg (DH) notation/method of placement for coordinate systems is used to determine the transformation matrices. The position measurement of the ball on the plate is realized using the camera system. As shown in Figure 10, with respect to the fixed location of the camera is necessary to transform the camera measured position of the ball to the actual position of the ball on the plate. Even in this case, the transformation matrix method is advantageously uses.

\footnotetext{
a e-mail: zatopek@fai.utb.cz
}

\section{Ball and plate}

\subsection{D model}

Figure 1 shows a complete 3D assembly model. This model is created in SolidWorks and has 4 generalized degrees of freedom. Tilts of the plate are two of them. They are realized by two rotary kinematic pairs in perpendicular relation to their common normal. The ball position on the plate are other two generalized degree of freedom.

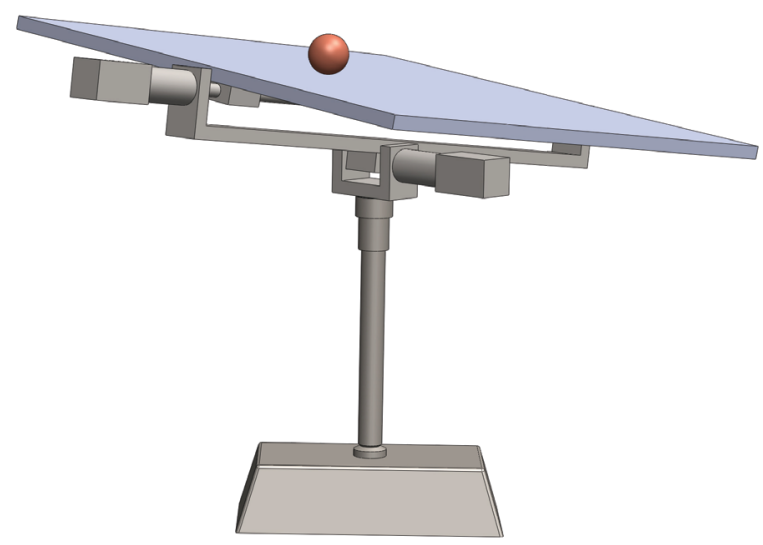

Figure 1. Total 3D model

For purposes of determining the system dynamic characteristics is preferably to simplify the system to the extent not too complicated equations of motion and at the same time the dynamic characteristics of the simplified model not be much different from the behaviour of the original system. 


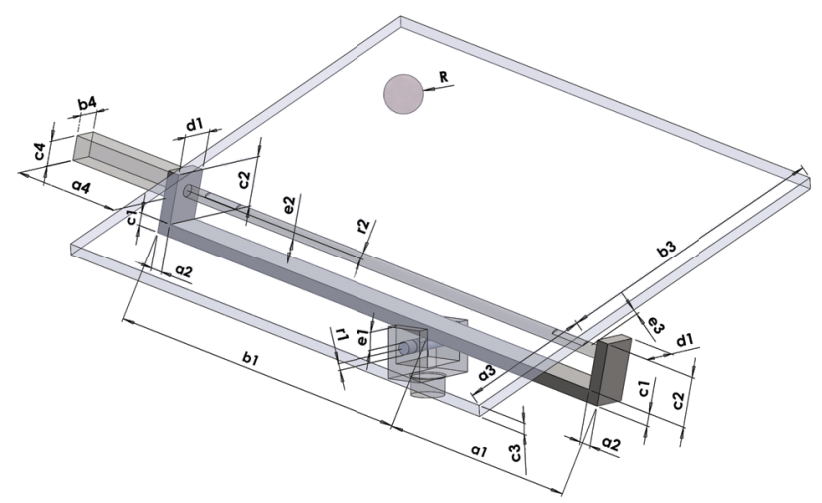

Figure 2. Simplified 3D model

The simplified model shown in Figure 2 contains only parts that have a major influence on its dynamics or serves as a global coordinate system base.

There are generally shown the basic dimensions that will be used in the coordinate system transformation - will be possible to change them in the resulting equation.

The stand as a base and the first motor need not be taken into account because they do not perform any movement (they are fixed) - as well as the connecting parts with negligible mass. The motor that moves the plate is replaced by cuboid.

\subsection{Transformation of coordinate systems}

The model is divided into seven parts for determining the homogeneous coordinates of the system. The part which is the transformation relates is always shown in the accompanying figures.

Used Denavit-Hartenberg (DH) notation/method of placement for coordinate systems is probably the most popular method used in robotics kinematics.

Table 1. DH parameters general table

\begin{tabular}{|l||l|l|l|l|}
\hline Link & $\boldsymbol{a}_{i}$ & $\boldsymbol{\alpha}_{i}$ & $\boldsymbol{\theta}_{i}$ & $\boldsymbol{d}_{i}$ \\
\hline \hline $\mathbf{1}$ & $a_{1}$ & $\alpha_{1}$ & $\theta_{1}$ & $d_{1}$ \\
\hline $\mathbf{2}$ & $a_{2}$ & $\alpha_{2}$ & $\theta_{2}$ & $d_{2}$ \\
\hline$\ldots$ & $\ldots$ & $\ldots$ & $\ldots$ & $\ldots$ \\
\hline $\mathbf{n}$ & $a_{n}$ & $\alpha_{n}$ & $\theta_{n}$ & $d_{n}$ \\
\hline
\end{tabular}

The calculation of the required transformations from the Table 1 is performed using the following relationships:

$$
\begin{gathered}
{ }^{\mathbf{i}-1} \mathbf{T}_{\mathbf{i}}=\left[\begin{array}{cccc}
\cos \theta_{i} & -\cos \alpha_{i} \sin \theta_{i} & \sin \alpha_{i} \sin \theta_{i} & a_{i} \cos \theta_{i} \\
\sin \theta_{i} & \cos \alpha_{i} \cos \theta_{i} & -\sin \alpha_{i} \cos \theta_{i} & a_{i} \sin \theta_{i} \\
0 & \sin \alpha_{i} & \cos \alpha_{i} & d_{i} \\
0 & 0 & 0 & 1
\end{array}\right] \\
{ }^{0} \mathbf{T}_{\mathbf{n}}={ }^{0} T_{1} \ldots{ }^{n-2} T_{n-1}{ }^{n-1} T_{n} \\
{ }^{\mathbf{G}} \mathbf{r}={ }^{G} T_{B} \cdot{ }^{B} r
\end{gathered}
$$

The ball transformation in a global coordinate system is listed here as a pattern, other transformations have similar characteristics. The global coordinate system $\left(X_{0}, Y_{0}, Z_{0}\right)$ is positioned at the rotation center axis of the first motor, as shown in Figure 3.

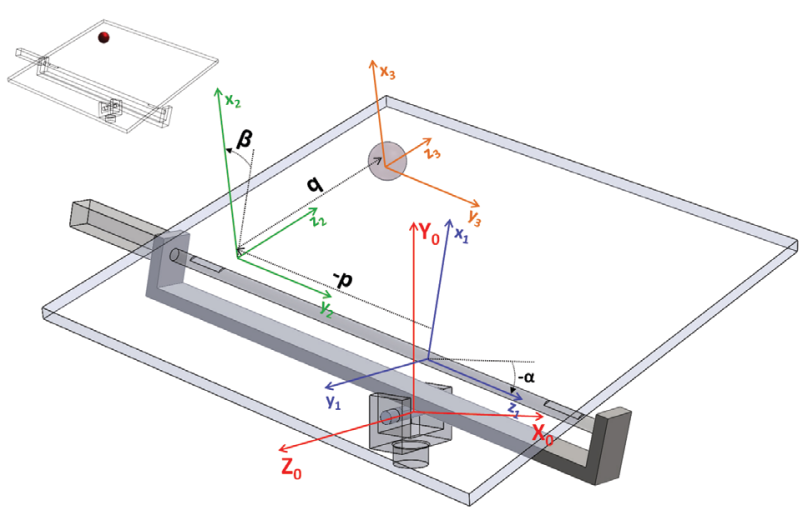

Figure 3. Coordinate system placement for the ball transformation - part 7

The gradual transformations from the Figure 3 can be written in Table 2. Utilising the relationship in the Equa-

Table 2. DH parameters for ball transformation - part 7

\begin{tabular}{|l||l|c|c|l|}
\hline Link & $\boldsymbol{a}_{i}$ & $\boldsymbol{\alpha}_{i}$ & $\boldsymbol{\theta}_{i}$ & $\boldsymbol{d}_{i}$ \\
\hline \hline $\mathbf{1}$ & $e_{1}+r_{1}+c_{1}+e_{2}+r_{2}$ & $\frac{\pi}{2}$ & $\frac{\pi}{2}$ & $\frac{\pi}{2}+\alpha$ \\
\hline $\mathbf{2}$ & $r_{2}+e_{3}+c_{3}+R$ & $\frac{\pi}{2}$ & $p$ & $\beta$ \\
\hline $\mathbf{3}$ & 0 & 0 & $q$ & 0 \\
\hline
\end{tabular}

tion 1 is found the transformation matrix between systems. Equation 2 is used for finding the final transformation matrix from the local body coordinate system - chosen at the center of gravity, into the global coordinate system. It is possible to determine the position vector ball in the global coordinate system (ball position in $3 \mathrm{D}$ space) by using the Equation 3.

The matrix ${ }^{0} T_{3}$ is listed in Equation 4. It is labelled as $T_{7}$ according to the selected assembly parts numbering.

$$
\mathbf{T}_{7}=\left[\begin{array}{cccc}
-\cos \beta \sin \alpha & \cos \alpha & -\sin \alpha \sin \beta & T_{7}^{1} \\
\cos \alpha \cos \beta & \sin \alpha & \cos \alpha \sin \beta & T_{7}^{2} \\
\sin \beta & 0 & -\cos \beta & T_{7}^{3} \\
0 & 0 & 0 & 1
\end{array}\right]
$$

in which:

$$
\begin{aligned}
T_{7}^{1}= & p \cos \alpha-\sin \alpha\left(c_{1}+e_{1}+e_{2}+r_{1}+r_{2}\right)- \\
& -q \sin \alpha \sin \beta-\cos \beta \sin \alpha\left(R+c_{3}+e_{3}+r_{2}\right) \\
T_{7}^{2}= & \cos \alpha\left(c_{1}+e_{1}+e_{2}+r_{1}+r_{2}\right)+p \sin \alpha+ \\
& +q \cos \alpha \sin \beta+\cos \alpha \cos \beta\left(R+c_{3}+e_{3}+r_{2}\right) \\
T_{7}^{3}= & \sin \beta\left(R+c_{3}+e_{3}+r_{2}\right)-q \cos \beta
\end{aligned}
$$

Transformation of the remaining parts of the assembly are given in Equations 5 - 10, Tables 3 - 8 and comes from Figure 4 - 9.

$$
\mathbf{T}_{\mathbf{1}}=\left[\begin{array}{cccc}
-\sin \alpha & 0 & \cos \alpha & T_{1}^{1} \\
\cos \alpha & 0 & \sin \alpha & T_{1}^{2} \\
0 & 1 & 0 & 0 \\
0 & 0 & 0 & 1
\end{array}\right]
$$




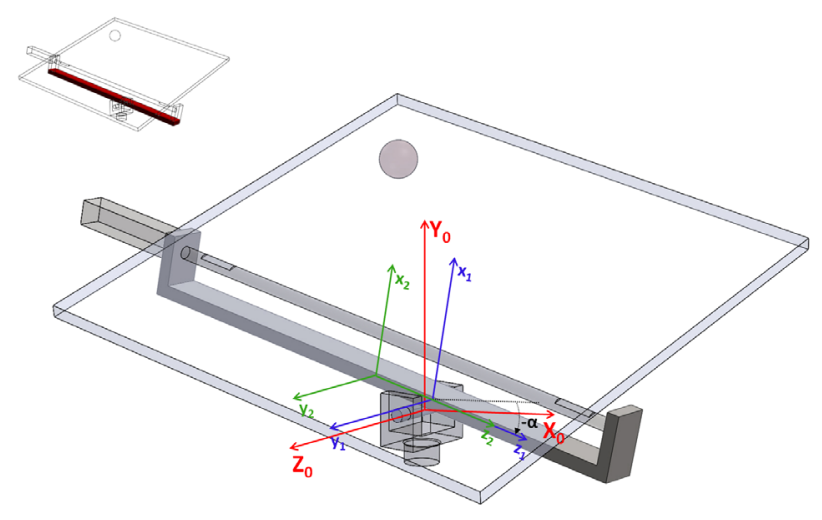

Figure 4. Coordinate system placement - part 1

Table 3. DH parameters - part 1

\begin{tabular}{|l||l|l|l|l|}
\hline Link & $\boldsymbol{a}_{i}$ & $\boldsymbol{\alpha}_{i}$ & $\boldsymbol{\theta}_{i}$ & $\boldsymbol{d}_{i}$ \\
\hline \hline $\mathbf{1}$ & $e_{1}+r_{1}+\frac{c_{1}}{2}$ & $\frac{\pi}{2}$ & 0 & $\frac{\pi}{2}+\alpha$ \\
\hline $\mathbf{2}$ & 0 & 0 & $\frac{a_{1}-b_{1}}{2}$ & 0 \\
\hline
\end{tabular}

in which:

$$
\begin{aligned}
& T_{1}^{1}=\cos \alpha \frac{a_{1}-b_{1}}{2}-\sin \alpha\left(\frac{c_{1}}{2}+e_{1}+r_{1}\right) \\
& T_{1}^{2}=\sin \alpha \frac{a_{1}-b_{1}}{2}+\cos \alpha\left(\frac{c_{1}}{2}+e_{1}+r_{1}\right)
\end{aligned}
$$

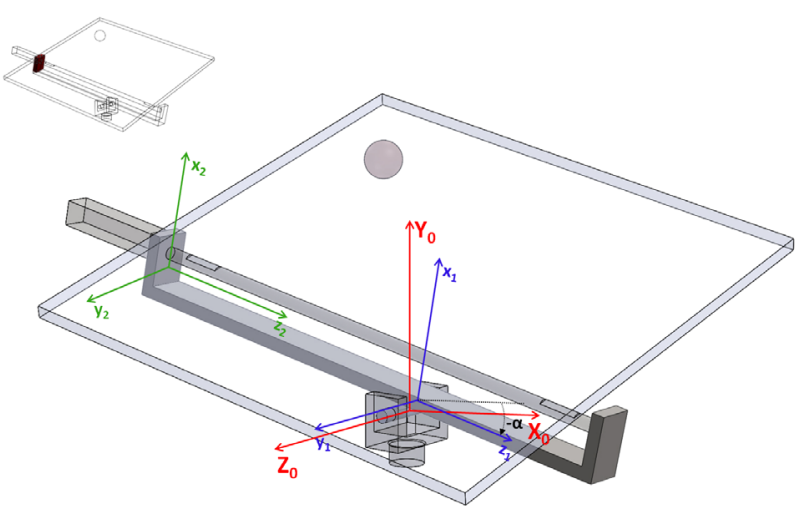

Figure 5. Coordinate system placement - part 2

Table 4. DH parameters - part 2

\begin{tabular}{|l||l|l|l|l|}
\hline Link & $\boldsymbol{a}_{i}$ & $\boldsymbol{\alpha}_{i}$ & $\boldsymbol{\theta}_{i}$ & $\boldsymbol{d}_{i}$ \\
\hline \hline $\mathbf{1}$ & $e_{1}+r_{1}+\frac{c_{1}}{2}$ & $\frac{\pi}{2}$ & 0 & $\frac{\pi}{2}+\alpha$ \\
\hline $\mathbf{2}$ & $\frac{c_{1}+c_{2}}{2}$ & 0 & $\frac{a_{2}}{2}-b_{1}$ & 0 \\
\hline
\end{tabular}

$$
\mathbf{T}_{2}=\left[\begin{array}{cccc}
-\sin \alpha & 0 & \cos \alpha & T_{2}^{1} \\
\cos \alpha & 0 & \sin \alpha & T_{2}^{2} \\
0 & 1 & 0 & 0 \\
0 & 0 & 0 & 1
\end{array}\right]
$$

in which:

$$
\begin{aligned}
& T_{2}^{1}=\cos \alpha\left(\frac{a_{1}}{2}-b_{1}\right)-\sin \alpha\left(c_{1}+\frac{c_{2}}{2}+e_{1}+r_{1}\right) \\
& T_{2}^{2}=\sin \alpha\left(\frac{a_{1}}{2}-b_{1}\right)+\cos \alpha\left(c_{1}+\frac{c_{2}}{2}+e_{1}+r_{1}\right)
\end{aligned}
$$

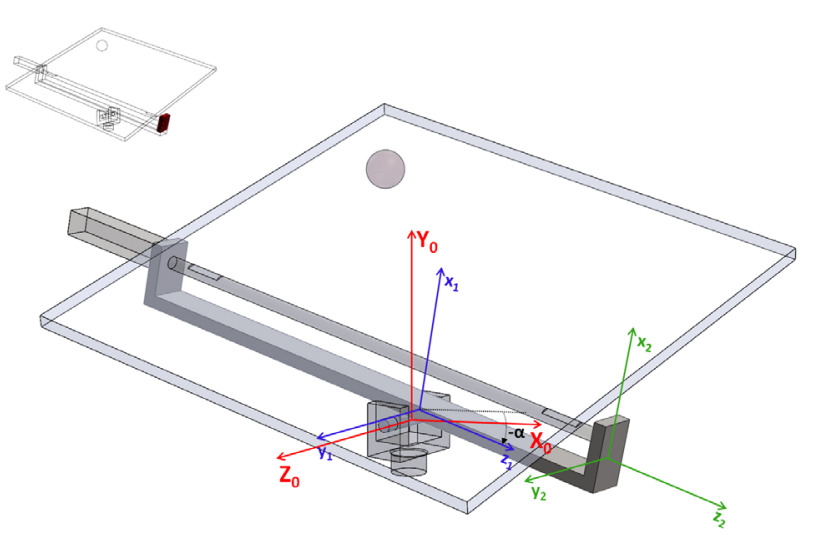

Figure 6. Coordinate system placement - part 3

Table 5. DH parameters - part 3

\begin{tabular}{|l||l|l|l|l|}
\hline Link & $\boldsymbol{a}_{i}$ & $\boldsymbol{\alpha}_{i}$ & $\boldsymbol{\theta}_{i}$ & $\boldsymbol{d}_{i}$ \\
\hline \hline $\mathbf{1}$ & $e_{1}+r_{1}+\frac{c_{1}}{2}$ & $\frac{\pi}{2}$ & 0 & $\frac{\pi}{2}+\alpha$ \\
\hline $\mathbf{2}$ & $\frac{c_{1}+c_{2}}{2}$ & 0 & $a_{1}-\frac{a_{2}}{2}$ & 0 \\
\hline
\end{tabular}

$$
\mathbf{T}_{\mathbf{3}}=\left[\begin{array}{cccc}
-\sin \alpha & 0 & \cos \alpha & T_{3}^{1} \\
\cos \alpha & 0 & \sin \alpha & T_{3}^{2} \\
0 & 1 & 0 & 0 \\
0 & 0 & 0 & 1
\end{array}\right]
$$

in which:

$$
\begin{aligned}
& T_{3}^{1}=\cos \alpha\left(a_{1}-\frac{a_{2}}{2}\right)-\sin \alpha\left(c_{1}+\frac{c_{2}}{2}+e_{1}+r_{1}\right) \\
& T_{3}^{2}=\sin \alpha\left(a_{1}-\frac{a_{2}}{2}\right)+\cos \alpha\left(c_{1}+\frac{c_{2}}{2}+e_{1}+r_{1}\right)
\end{aligned}
$$

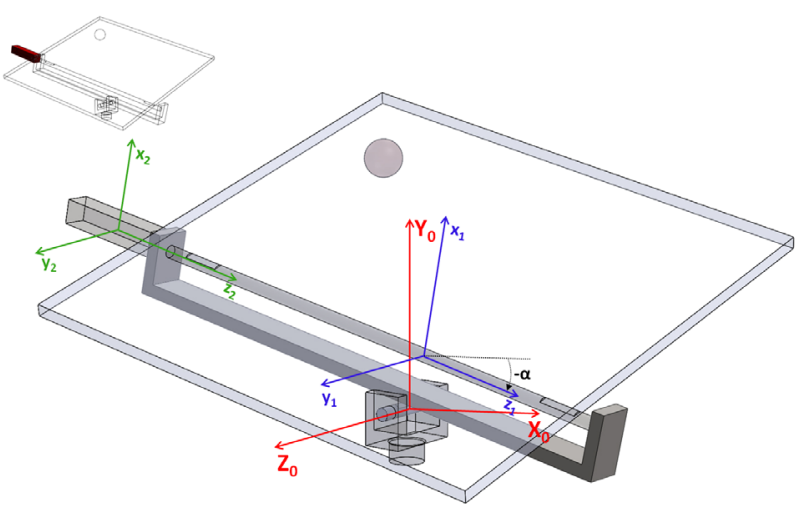

Figure 7. Coordinate system placement - part 4

Table 6. DH parameters - part 4

\begin{tabular}{|l||l|l|l|l|}
\hline Link & $\boldsymbol{a}_{i}$ & $\boldsymbol{\alpha}_{i}$ & $\boldsymbol{\theta}_{i}$ & $\boldsymbol{d}_{i}$ \\
\hline \hline $\mathbf{1}$ & $e_{1}+r_{1}+c_{1}+e_{2}+r_{2}$ & $\frac{\pi}{2}$ & 0 & $\frac{\pi}{2}+\alpha$ \\
\hline $\mathbf{2}$ & 0 & 0 & $-b_{1}-\frac{a_{4}}{2}$ & 0 \\
\hline
\end{tabular}




$$
\mathbf{T}_{4}=\left[\begin{array}{cccc}
-\sin \alpha & 0 & \cos \alpha & T_{4}^{1} \\
\cos \alpha & 0 & \sin \alpha & T_{4}^{2} \\
0 & 1 & 0 & 0 \\
0 & 0 & 0 & 1
\end{array}\right]
$$

in which:

$$
\begin{aligned}
& T_{4}^{1}=-\sin \alpha\left(c_{1}+e_{1}+r_{1}+e_{2}+r_{2}\right)-\cos \alpha\left(b_{1}+\frac{a_{4}}{2}\right) \\
& T_{4}^{2}=\cos \alpha\left(c_{1}+e_{1}+r_{1}+e_{2}+r_{2}\right)-\sin \alpha\left(b_{1}+\frac{a_{4}}{2}\right)
\end{aligned}
$$

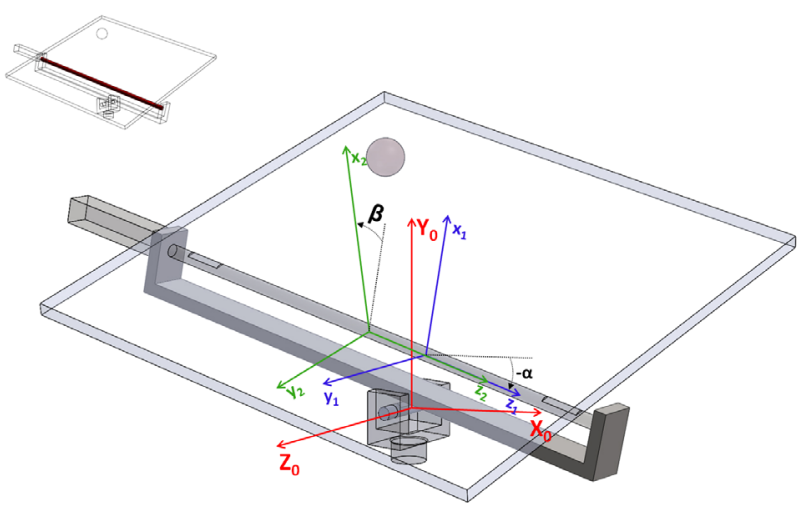

Figure 8. Coordinate system placement - part 5

Table 7. DH parameters - part 5

\begin{tabular}{|l||l|l|l|l|}
\hline Link & $\boldsymbol{a}_{i}$ & $\boldsymbol{\alpha}_{i}$ & $\boldsymbol{\theta}_{i}$ & $\boldsymbol{d}_{i}$ \\
\hline \hline $\mathbf{1}$ & $e_{1}+r_{1}+c_{1}+e_{2}+r_{2}$ & $\frac{\pi}{2}$ & 0 & $\frac{\pi}{2}+\alpha$ \\
\hline $\mathbf{2}$ & 0 & 0 & $\frac{a_{1}-b_{1}}{2}$ & $\beta$ \\
\hline
\end{tabular}

$$
\mathbf{T}_{\mathbf{5}}=\left[\begin{array}{cccc}
-\cos \beta \sin \alpha & \sin \alpha \sin \beta & \cos \alpha & T_{5}^{1} \\
\cos \alpha \cos \beta & -\cos \alpha \sin \beta & \sin \alpha & T_{5}^{2} \\
\sin \beta & \cos \beta & 0 & 0 \\
0 & 0 & 0 & 1
\end{array}\right]
$$

in which:

$$
\begin{aligned}
& T_{5}^{1}=\cos \alpha \frac{a_{1}-b_{1}}{2}-\sin \alpha\left(c_{1}+e_{1}+r_{1}+e_{2}+r_{2}\right) \\
& T_{5}^{2}=\sin \alpha \frac{a_{1}-b_{1}}{2}+\cos \alpha\left(c_{1}+e_{1}+r_{1}+e_{2}+r_{2}\right)
\end{aligned}
$$

Table 8. DH parameters - part 6

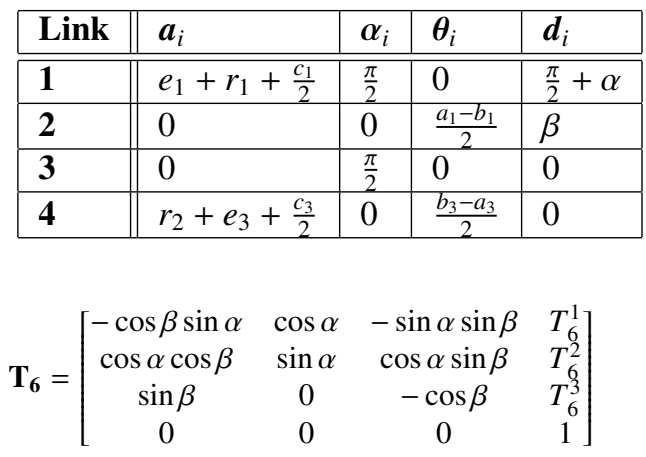

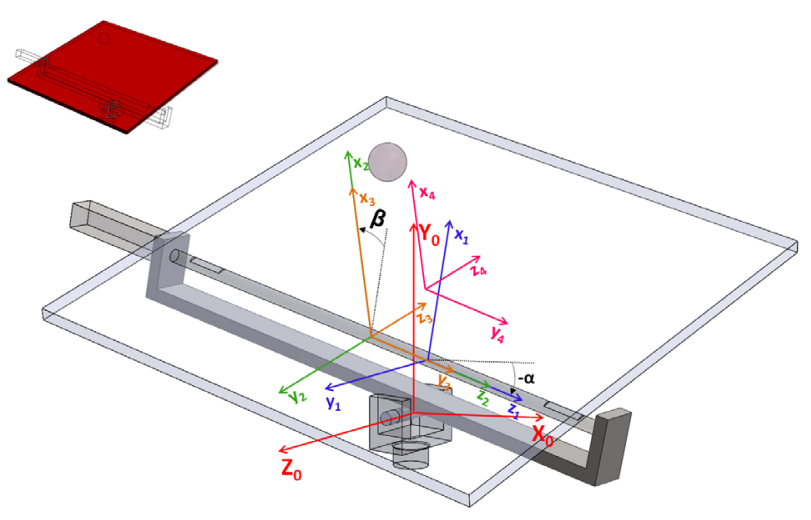

Figure 9. Coordinate system placement - part 6

in which:

$$
\begin{aligned}
T_{6}^{1}= & \cos \alpha \frac{a_{1}-b_{1}}{2}-\sin \alpha\left(c_{1}+e_{1}+r_{1}+e_{2}+r_{2}\right)+ \\
& +\sin \alpha \sin \beta \frac{a_{3}-b_{3}}{2}-\cos \beta \sin \alpha\left(r_{2}+e_{3}+\frac{c_{3}}{2}\right) \\
T_{6}^{2}= & \sin \alpha \frac{a_{1}-b_{1}}{2}+\cos \alpha\left(c_{1}+e_{1}+r_{1}+e_{2}+r_{2}\right)- \\
& -\cos \alpha \sin \beta \frac{a_{3}-b_{3}}{2}+\cos \alpha \cos \beta\left(r_{2}+e_{3}+\frac{c_{3}}{2}\right) \\
T_{6}^{3}= & \cos \beta \frac{a_{3}-b_{3}}{2}+\sin \beta\left(r_{2}+e_{3}+\frac{c_{3}}{2}\right)
\end{aligned}
$$

\subsection{Motion Equations}

Motion equations matrix form of serial manipulator has the general form:

$$
\mathrm{D}(\overline{\mathbf{q}}) \cdot \ddot{\overrightarrow{\mathbf{q}}}+\overline{\mathbf{H}}(\overline{\mathbf{q}}, \dot{\overline{\mathbf{q}}})+\overline{\mathbf{G}}(\overline{\mathbf{q}})=\overline{\mathbf{Q}}
$$

in which:

$\mathrm{D}(\overline{\mathbf{q}})$... symmetric inertia matrix

$\overline{\mathbf{H}}(\overline{\mathbf{q}}, \dot{\overline{\mathbf{q}}})$... bounded velocity vector

$\overline{\mathbf{G}}(\overline{\mathbf{q}})$... gravitational force vector

$\overline{\mathbf{Q}}$...nnon-potential (non-conservative) generalized forces causing change of $\overline{\mathbf{q}}$

Individual parts of the Equation 11 can be decomposed to the form:

$$
\mathbf{D}_{\mathbf{i}, \mathbf{j}}=\sum_{r=\max \{i, j\}}^{n} \operatorname{tr}\left[\frac{\partial^{0} T_{r}}{\partial q_{j}} \cdot{ }^{r} \bar{I}_{r}\left(\frac{\partial^{0} T_{r}}{\partial q_{i}}\right)^{T}\right]
$$

$$
\begin{gathered}
\mathbf{H}_{\mathbf{i}}=\sum_{k=1}^{n} \sum_{m=1}^{n} H_{i k m} \dot{q}_{k} \dot{q}_{m} \\
\mathbf{H}_{\mathbf{i k m}}=\sum_{r=\max \{i, k, m\}}^{n} \operatorname{tr}\left[\frac{\partial^{2}{ }^{0} T_{r}}{\partial q_{k} \partial q_{m}} \cdot{ }^{r} \bar{I}_{r}\left(\frac{\partial^{0} T_{r}}{\partial q_{i}}\right)^{T}\right] \\
\mathbf{G}_{\mathbf{i}}=-\sum_{r=i}^{n} m_{r}\left({ }^{0} \bar{g}\right)^{T} \cdot \frac{\partial^{0} T_{r}}{\partial q_{i}} \cdot{ }^{r} \bar{r}_{r}
\end{gathered}
$$

in which:

$n \ldots$ number of links

$i, j \ldots$ state variables 
$\bar{I}$...pseudo-inertia matrix

${ }^{0} \bar{g}$... gravitational vector in global coordinate system

${ }^{r} \bar{r}_{r} \ldots$ the center of mass position vector of link in the local coordinate system

The gravitational vector ${ }^{0} \bar{g}$ is in the direction of the global axis $Y$, therefore:

$$
\mathbf{0} \overline{\mathbf{g}}=\left[\begin{array}{c}
0 \\
-g \\
0 \\
1
\end{array}\right]
$$

The center of mass position vector of link is zero in his local coordinate system - all the local coordinate systems are placed in the body center of mass:

$$
\mathbf{r}_{\mathbf{r}_{\mathbf{r}}}=\left[\begin{array}{c}
r_{x} \\
r_{y} \\
r_{z} \\
1
\end{array}\right]=\left[\begin{array}{l}
0 \\
0 \\
0 \\
1
\end{array}\right]
$$

Only the last unknown parameter is missing in Equation 11 - the pseudo-inertia matrix $\bar{I}$.

\subsubsection{Inertia matrix}

The inertia matrix of individual assembly parts is also need to know to derive the pseudo-inertia matrices in their local coordinate system. Because the local coordinate system are always placed in the object center of mass the deviance moments (i.e. off-diagonal elements) of inertia matrices will be zero.

The numbering is similar as for the transformation matrices.

$$
\begin{aligned}
& \mathbf{I}_{1}=\frac{m_{1}}{12}\left[\begin{array}{ccc}
\left(a_{1}+b_{1}\right)^{2}+d_{1}^{2} & 0 & 0 \\
0 & \left(a_{1}+b_{1}\right)^{2}+c_{1}^{2} & 0 \\
0 & 0 & c_{1}^{2}+d_{1}^{2}
\end{array}\right] \\
& \mathbf{I}_{\mathbf{2}}=\mathbf{I}_{\mathbf{3}}=\frac{m_{2}}{12}\left[\begin{array}{ccc}
a_{2}^{2}+d_{1}^{2} & 0 & 0 \\
0 & a_{2}^{2}+c_{2}^{2} & 0 \\
0 & 0 & c_{2}^{2}+d_{1}^{2}
\end{array}\right] \\
& \mathbf{I}_{\mathbf{4}}=\frac{m_{4}}{12}\left[\begin{array}{ccc}
a_{4}^{2}+b_{4}^{2} & 0 & 0 \\
0 & a_{4}^{2}+c_{4}^{2} & 0 \\
0 & 0 & b_{4}^{2}+c_{4}^{2}
\end{array}\right] \\
& \mathbf{I}_{5}=\frac{m_{5}}{12}\left[\begin{array}{ccc}
3 r_{2}^{2}+\left(a_{1}+b_{1}-2 a_{2}\right)^{2} & 0 & 0 \\
0 & 3 r_{2}^{2}+\left(a_{1}+b_{1}-2 a_{2}\right)^{2} & 0 \\
0 & 0 & r_{2}^{2}
\end{array}\right] \\
& \mathbf{I}_{\mathbf{6}}=\frac{m_{6}}{12}\left[\begin{array}{ccc}
\left(a_{3}+b_{3}\right)^{2}+d_{3}^{2} & 0 & 0 \\
0 & \left(a_{3}+b_{3}\right)^{2}+c_{3}^{2} & 0 \\
0 & 0 & c_{3}^{2}+d_{3}^{2}
\end{array}\right] \\
& \mathbf{I}_{7}=\frac{2}{5} m_{7} R^{2}\left[\begin{array}{ccc}
1 & 0 & 0 \\
0 & 1 & 0 \\
0 & 0 & 1
\end{array}\right]
\end{aligned}
$$

\subsubsection{Pseudo-inertia matrix}

$$
\overline{\mathbf{I}}=\left[\begin{array}{cccc}
\frac{-I_{x x}+I_{y y}+I_{z z}}{2} & I_{x y} & I_{x z} & m r_{x} \\
I_{y x} & \frac{I_{x x}-I_{y y}+I_{z z}}{2} & I_{y z} & m r_{y} \\
I_{z x} & I_{z y} & \frac{I_{x x}+I_{y y}-I_{z z}}{2} & m r_{z} \\
m r_{x} & m r_{y} & m r_{z} & m
\end{array}\right]
$$

The general definition of pseudo-inertia matrix is listed in Equation 24. Now there is all you need to derive the resulting motion equations - Equation 11.

$$
\begin{aligned}
& \mathbf{I}_{1 \mathbf{P}}=\left[\begin{array}{cccc}
\frac{m_{1} c_{1}^{2}}{12} & 0 & 0 & 0 \\
0 & \frac{m_{1} d_{1}^{2}}{12} & 0 & 0 \\
0 & 0 & \frac{m_{1}\left(a_{1}+b_{1}\right)^{2}}{12} & 0 \\
0 & 0 & 0 & m_{1}
\end{array}\right] \\
& \mathbf{I}_{\mathbf{2}} \mathbf{P}=\mathbf{I}_{\mathbf{3 P}}=\left[\begin{array}{cccc}
\frac{m_{2} c_{2}^{2}}{12} & 0 & 0 & 0 \\
0 & \frac{m_{2} d_{1}^{2}}{12} & 0 & 0 \\
0 & 0 & \frac{m_{2} a_{2}^{2}}{12} & 0 \\
0 & 0 & 0 & m_{2}
\end{array}\right] \\
& \mathbf{I}_{4 \mathbf{P}}=\left[\begin{array}{cccc}
\frac{m_{4} c_{4}^{2}}{12} & 0 & 0 & 0 \\
0 & \frac{m_{4} b_{4}^{2}}{12} & 0 & 0 \\
0 & 0 & \frac{m_{4} a_{4}^{2}}{12} & 0 \\
0 & 0 & 0 & m_{4}
\end{array}\right] \\
& \mathbf{I}_{5 \mathbf{P}}=\left[\begin{array}{cccc}
\frac{m_{5} r_{2}^{2}}{4} & 0 & 0 & 0 \\
0 & \frac{m_{5} r_{2}^{2}}{4} & 0 & 0 \\
0 & 0 & \frac{m_{5}\left(a_{1}-2 a_{2}+b_{1}\right)^{2}}{12} & 0 \\
0 & 0 & 0 & m_{5}
\end{array}\right] \\
& \mathbf{I}_{6} \mathbf{P}=\left[\begin{array}{cccc}
\frac{m_{6} c_{3}^{2}}{12} & 0 & 0 & 0 \\
0 & \frac{m_{6} d_{3}^{2}}{12} & 0 & 0 \\
0 & 0 & \frac{m_{6}\left(a_{3}+b_{3}\right)^{2}}{12} & 0 \\
0 & 0 & 0 & m_{6}
\end{array}\right] \\
& \mathbf{I}_{7 \mathbf{P}}=\left[\begin{array}{cccc}
\frac{m_{7} R^{2}}{5} & 0 & 0 & 0 \\
0 & \frac{m_{7} R^{2}}{5} & 0 & 0 \\
0 & 0 & \frac{m_{7} R^{2}}{5} & 0 \\
0 & 0 & 0 & m_{7}
\end{array}\right]
\end{aligned}
$$

\subsection{The ball position on the plate}

Obviously, the transformation matrix using is not only refer to the motion equations. The system has four generalized degree of freedom as Section 2.1 shows. This state variable is need to know during all the regulation process.

Tilts of the plate are given by encoders on the motors which controls them. However, the ball position on the plate is unknown - it rolls independently in the direction of the fastest decrease in potential energy - a negative gradient. It is given by the tilt of the plane.

The ball position on the plate is measured by using a camera, which is placed as shown in Figure 10 - the optical axis of the camera is identical to the global axis $Y_{0}$ and the plate edges are parallel to the camera pixel array edges. 
The ball coordinates on axes $X_{0}$ and $Z_{0}$ are measured by this placement, not the size of the $p$ and $q$ as shown in Figure 3.

The best way would be moving the camera and the plate together, but it is ineffective from the construction and control viewpoint. However, the $p$ and $q$ parameters is possible to calculate with the transformation matrices help.

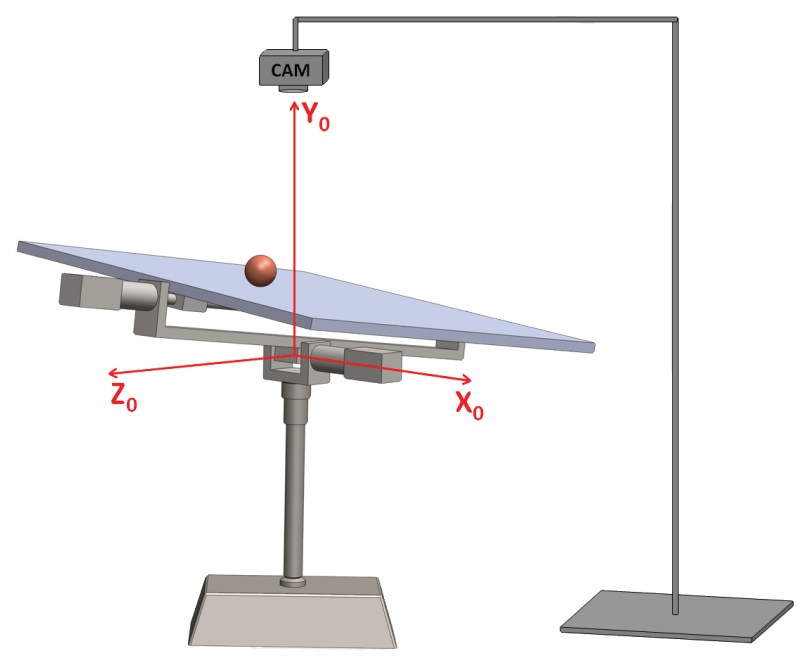

Figure 10. 3D Model with camera evaluation

The ball position in the global coordinate system is expressed from Equation 3.

$$
\begin{aligned}
\mathbf{X}= & p \cos \alpha-\sin \alpha\left(c_{1}+e_{1}+e_{2}+r_{1}+r_{2}\right)- \\
& -q \sin \alpha \sin \beta-\cos \beta \sin \alpha\left(R+c_{3}+e_{3}+r_{2}\right) \\
\mathbf{Y}= & \cos \alpha\left(c_{1}+e_{1}+e_{2}+r_{1}+r_{2}\right)+p \sin \alpha+ \\
& +q \cos \alpha \sin \beta+\cos \alpha \cos \beta\left(R+c_{3}+e_{3}+r_{2}\right) \\
\mathbf{Z =}= & \sin \beta\left(R+c_{3}+e_{3}+r_{2}\right)-q \cos \beta
\end{aligned}
$$

Parameters $p$ and $q$ are expressed from Equation 31, $X$ and $Z$ are measured by the camera system.

$$
\begin{gathered}
X-\frac{\sin \alpha \sin \beta\left[Z-\sin \beta\left(R+c_{3}+e_{3}+r_{2}\right)\right]}{\cos \beta}+ \\
\mathbf{p}=\frac{\begin{array}{c}
+\sin \alpha\left(c_{1}+e_{1}+e_{2}+r_{1}+r_{2}\right)+ \\
+\cos \beta \sin \alpha\left(R+c_{3}+e_{3}+r_{2}\right)
\end{array}}{\cos \alpha} \\
\mathbf{q}=\frac{\sin \beta\left(R+c_{3}+e_{3}+r_{2}\right)-Z}{\cos \beta}
\end{gathered}
$$

All the state variables are already fully defined. If the vertical ball position is necessary to know in the global coordinate system, state variables $p$ and $q$ just put into $Y$ in Equation 31.

\section{Conclusion}

The article contains derivations of the transformation, inertia matrices and all other required formalities neces- sary for the determine motion equations using the matrix form shown in Equation11. The substitution into the Equation 11 and the resultant form is not listed here due to the high dimensionality of the resulting expression. This substitutions and mathematics adjustments are implemented in MATLAB.

The model is divided into 7 parts for ease identifying and acceptable inertia matrices form - each part is one of the basic shapes. These parts connection occurs during substituting into the Equation 11.Transformation matrices are designed to transform the local coordinate system position, associated with the object center of mass, to the global coordinate system. This transformation ensures zero inertia matrix deviance moments and therefore zero pseudo-inertia matrices off-diagonal elements.

Transformation matrices are also used in the regulated value determining, which is different from the actual values, with respect to the fixed camera location to the moving plate. It is used for conversion between the measured and the actual output/regulated value.

Suitable coordinate system placement and then the transformation matrix form is a key consideration as follows from the foregoing. It is important from the viewpoint of complexity of other calculations, from the perspective of finding potential errors or their further use correctness of the transformation matrix can be easily geometrically verified, which can not be said about the system dynamics describing structures.

\section{References}

[1] S. Crandall, D. C, .Karnopp, E.F. Kurz, Dynamics of mechanical and electromechanical systems (McGrawHill, NewYork, 1968) 466

[2] H. K. Khali, Nonlinear Systems (Upper Saddle River, N.J: Pearson, 2001) 750

[3] M.-T. Ho, Y. Rizal, L.-M. Chu, International Journal of Advanced Robotic Systems \& Exposition 10, 16 (2013)

[4] M. Nokhbeh, D. Khashabi, H. A. Talebi, Final Project Report from Amirkabir University of Technology 1, 22 (2011)

[5] Z. Úředníček, Robotika (Zlín, T. Bata university in Zlin, 2012) 284

[6] Z. Úředníček, Elektromechanické akční členy (Zlín, T. Bata university in Zlin, 2009) 127

[7] D. C. Karnopp, R. C. Rosenberg, System dynamics (A Unifies Approach. Wiley \& Sons, New York, 1975) 372

[8] X. Dong, Y. Zhao, Y. Xu, Z. Zhang, P. Shi, Internacional Journal of Innovative Computing, Information and Control 7, 13 (2011) 